\title{
Long-range corrected DFT calculations of charge-transfer integrals in model metal-free phthalocyanine complexes
}

\author{
Mikolaj M. Mikolajczyk • Robert Zaleśny • \\ Żaneta Czyżnikowska • Petr Toman • \\ Jerzy Leszczynski • Wojciech Bartkowiak
}

Received: 30 July 2010 /Accepted: 29 September 2010 / Published online: 27 October 2010

(C) The Author(s) 2010. This article is published with open access at Springerlink.com

\begin{abstract}
An assessment of several widely used exchangecorrelation potentials in computing charge-transfer integrals is performed. In particular, we employ the recently proposed Coulomb-attenuated model which was proven by other authors to improve upon conventional functionals in the case of charge-transfer excitations. For further validation, two distinct approaches to compute the property in question are compared for a phthalocyanine dimer.
\end{abstract}

Keywords Charge-transfer integral · Density functional theory · Long-range corrected functionals · Organic electronics $\cdot$ Phthalocyanine

\footnotetext{
M. M. Mikolajczyk $\cdot$ R. Zaleśny $\cdot$ Ż. Czyżnikowska $\cdot$

W. Bartkowiak $(\bowtie)$

Theoretical Chemistry Group, Institute of Physical and Theoretical Chemistry, Wroclaw University of Technology, Wybrzeże Wyspiańskiego 27,

50-370 Wrocław, Poland

e-mail: wojciech.bartkowiak@pwr.wroc.pl

P. Toman

Institute of Macromolecular Chemistry,

Academy of Sciences of the Czech Republic,

v.v.i., Heyrovský Sq. 2,

16206 Prague 6, Czech Republic

Ż. Czyżnikowska

Department of Inorganic Chemistry, Faculty of Pharmacy,

Wroclaw Medical University,

Szewska 38,

50-139 Wrocław, Poland

R. Zaleśny $\cdot$ J. Leszczynski

Interdisciplinary Center for Nanotoxicity,

Department of Chemistry, Jackson State University,

Jr Lynch St. 1400,

Jackson, MS 39217, USA
}

\section{Introduction}

The charge-transfer integral is an essential parameter in several theoretical models describing charge carrier transport in organic materials [1-7]. It is very often assumed that charge carrier mobility is proportional to the square of the charge-transfer integral $(J)$ which describes the transport of a charge between adjacent molecular sites. An inherent issue of practical computations of charge-transfer integrals represents the choice of an approach to solve the Schrödinger equation. Currently, the DFT framework is commonly used to model charge transport in organic materials [3, 8-14]. Certain exchange-correlation potentials are recognized to predict accurately geometries of molecules and shapes of molecular orbitals. However, it is well known, that wrong asymptotic behavior of conventional functionals create a real problem in calculations of some properties, especially for molecular complexes $[15,16]$. A recent systematic study of Peach and co-workers may serve as an illustrative example [17]. The authors showed that conventional exchangecorrelation functionals have difficulties with reliable description of excitation energies to charge-transfer states in molecules and molecular complexes. The charge-transfer integral $(J)$ involves orbitals localized on the two adjacent sites. For this reason, its evaluation might also present a challenge for the conventional exchange-correlation potentials commonly used nowadays. The primary goal of this study is to shed some light on this issue by employing recently proposed long-range corrected density functional theory (hereafter denoted as LRC-DFT) to compute chargetransfer integrals. The LRC-DFT is still being extensively tested primarily with an eye toward electric dipole (hyper) polarizabilities, linear and nonlinear optical spectra [17-29]. Here, we use two LRC functionals, namely LC-BLYP [30] and CAM-B3LYP [31] together with their conventional 
counterparts. The LRC functionals employ the Ewald split of $r_{12}^{-1}$ operator which, in the case of the CAM-B3LYP functional, takes the following form [31]:

$\frac{1}{r_{12}}=\frac{1-\left[\alpha+\beta \cdot \operatorname{erf}\left(\mu r_{12}\right)\right]}{r_{12}}+\frac{\alpha+\beta \cdot \operatorname{erf}\left(\mu r_{12}\right)}{r_{12}}$,

where the first and the second term on the rhs of the equation account for the short- and the long-range interactions, respectively, and $\alpha$ and $\beta$ are constants. Here, we use the models based on the above equation with $\mu=0.33$ for CAMB3LYP and $\mu=0.47$ for LC-BLYP [32].

As a model system to evaluate the performance of conventional exchange-correlation potentials in computing charge-transfer integrals we have chosen metal-free phthalocyanine dimer. Phthalocyanines are often considered as conductive materials with potential applications in organic electronics [33-36]. In crystalline phase phthalocyanine molecules usually form regular columns and liquid crystals composed of phthalocyanines are promising materials for organic electronics [37]. The liquid crystals in question are usually built from flat aromatic phthalocyanine center and aliphatic side groups. Likewise, aromatic core of molecules in liquid crystal state form regular columns with molecules in stacked conformations and the fastest charge transport is observed inside a column with much smaller probability of charge transport between columns. The charge-transfer integral between monomers in dimer can be used to describe charge transport inside of column composed of phthalocyanine molecules and as a first approximation of charge-transfer in phthalocyanine based liquid crystals. In this work only chargetransfer integrals between highest occupied molecular orbitals (HOMOs) of adjacent monomers are considered. This represents the charge-transfer integral related to the transport of positive charge carrier (hole transport).

\section{Computational details}

The initial structure of the dimer was composed of two phthalocyanine monomers in stacked conformation with rotation axis passing through the centers of mass of monomers and perpendicular to the plane of monomer. The structure of the phthalocyanine monomer was optimized at the B3LYP/6-31G(d) level of theory and was not reoptimized in dimer. The configurational space was spanned by a set of three parameters, namely intermolecular distance, twist angle around the symmetry axis and lateral slide of one of the monomers in two directions (see Fig. 1). What follows is a brief description of techniques used to compute charge-transfer integrals which are defined as:

$J_{s, s^{\prime}}=\left\langle\psi_{s}\left|h_{K S}\right| \psi_{s^{\prime}}\right\rangle$,
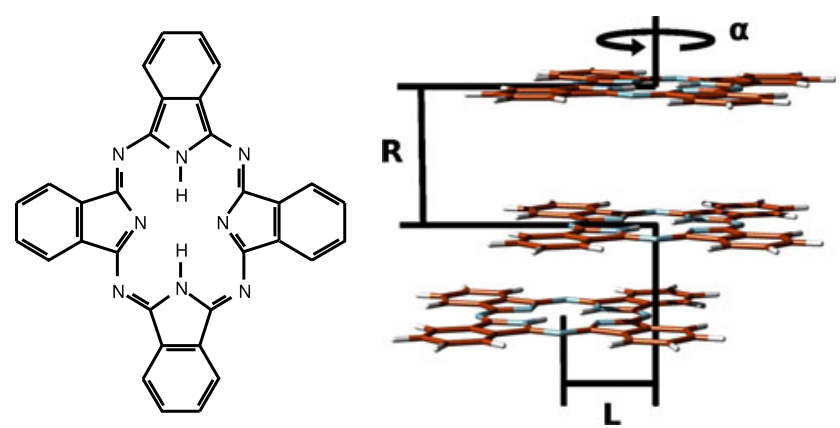

Fig. 1 The schematic representation of three geometrical parameters used to describe the structure of phthalocyanine dimer: $\alpha$ denotes twist angle, $\mathbf{R}$ is intermolecular distance and $\mathbf{L}$ stands for lateral slide

where $h_{K S}$ is the Kohn-Sham Hamiltonian, $\Psi s, \Psi s^{\prime}$ denote wave functions of the charge carrier localized on the sites $s$ and $\mathrm{s}^{\prime}$, respectively. In many organic systems there is non-zero spatial overlap between orbitals of the molecular sites. To account for this effect in calculations of charge-transfer integrals, the effective charge-transfer integral $\left(J_{\text {eff }}\right)$ may be introduced [4]:

$J_{e f f}=J_{s, s^{\prime}}-\frac{1}{2} S_{s, s^{\prime}}\left(\varepsilon_{s}+\varepsilon_{s^{\prime}}\right)$

$S_{s, s^{\prime}}$ denotes overlap integral of orbitals $s$ and $s^{\prime} ; \varepsilon_{s}$ and $\varepsilon_{s^{\prime}}$ stand for energies of the sites $s$ and $s^{\prime}$, respectively, and hereafter will be referred to as site energies.

Equation 2 can be directly used to compute the chargetransfer integral. In doing so, we express the Hamiltonian of the dimer $\left(h_{K S}\right)$ in the basis of molecular orbitals of the monomers $[3,38]$ :

$h_{K S}=S C E C^{-1}$.

In order to compute the charge-transfer integral one needs to determine the eigenvalues for a dimer $(E)$, the eigenvectors in the basis of atomic orbitals $(\mathrm{AO})$ for a dimer $\left(C_{A O}\right)$, the spatial overlap integrals in AO representation for a dimer $\left(S_{A O}\right)$ and eigenvectors for monomers in $\mathrm{AO}$ representation $\left(C_{A O}^{\prime}\right.$ and $\left.C_{A O}^{\prime \prime}\right)$. The eigenvector matrix for a dimer and spatial overlap matrix was transformed from $\mathrm{AO}$ representation to molecular orbital representation of monomers as follows:

$S=A S_{A O} A^{T}$,

$C=A C_{A O} A^{T}$,

where $A$ denotes transformation matrix which is diagonal block matrix with monomer eigenvector matrices $\left(C_{A O}^{\prime}\right.$ and $\left.C_{A O}^{\prime \prime}\right)$ on the diagonal and $A^{T}$ is transposed transformation matrix. The off-diagonal elements of $h_{K S}$ 
matrix in monomer orbital basis represent charge-transfer integrals.

Another approach to compute the charge-transfer integrals, even more frequently used than the one described above, is the method which introduces energy splitting in dimer [39-41]. In this approach, the eigenvalue of sth molecular orbital of the Hamiltonian for dimer is given by:

$E_{s}=\frac{\alpha_{s} \pm J_{s}}{1 \pm S_{s}}$

where $\alpha_{s}$ is the $s$-th site energy integral, $J_{s}$ and $S_{s}$ are the charge-transfer and the overlap integral between orbitals denoted by index $s$ of two molecules forming dimer, respectively. It is assumed that the mixing between $s$-th orbital and the other molecular orbitals is not significant. The splitting in energy of $s$-th molecular orbital of monomer in a dimer can be written as:

$\left|E_{s, A}-E_{s, B}\right|=\left|\frac{2 J_{s}-2 S_{S} \alpha_{s}}{1-S_{s}^{2}}\right|$.

In Eq. (8) $E_{s, A}$ and $E_{s, B}$ denote eigenvalues for $s$-th molecular orbital of molecules A and B, respectively. $J_{s}, \alpha_{s}, S_{s}$ stand for the charge-transfer integral, site energy and overlap integral of $s$-th molecular orbital of molecules A and B, respectively. Since we consider a dimer composed of two identical monomers, it is further assumed that $\alpha_{s}$ is the same for monomers A and B. For a system composed of two nonequivalent molecules, expressions for the eigenvalue $E_{s}$ and the charge transfer integral $J_{s}$ take more complicated form [41]. Usually, it is also assumed that spatial overlap integral is equal zero $\left(S_{s}=0\right)$, which is reasonable assumption considering charge transport between two organic molecules. In organic materials spatial overlap between orbitals of neighboring molecules are usually $<<1$. Thus, Eq. (8) can be rewritten as:

$\frac{1}{2}\left|E_{S, A}-E_{S, B}\right| \equiv \Delta / 2=\left|J_{S}\right|$.

In the present study we compute charge-transfer integrals using both above described methods. Therefore, once the splitting in energy is known, the charge-transfer integral $J$ can be calculated according to this relation.

Calculations were performed with the aid of several exchange-correlation potentials using different basis sets, including Dunning's correlation consistent ccpVDZ basis set [42] as well as recently proposed Jensen's basis set [43]. The results of calculations presented in this work were carried out using the GAUSSIAN 09 program [32].

\section{Results and discussion}

Among several factors responsible for accuracy of results of computations of charge-transfer integrals one should not overlook the choice of the basis set. Most quantumchemistry programs use the Gaussian functions, introduced by Boys in the mid 1950s, to approximate a wave function. Huang and Kertesz recently made an attempt to analyze the charge-transfer integrals using various basis sets and proved that basis set limit might be reached quite rapidly for the property in question [40]. It should be underlined that the smooth convergence of charge-transfer integrals with the basis set size is observed for effective chargetransfer integrals or in the case of negligible spatial overlap. Since the phthalocyanine complexes studied in the present work do not fulfill the latter criteria, we find it interesting to explore this topic more deeply by including the spatial overlap in computations of charge-transfer integrals. In Fig. 2a the values of spatial overlap integrals, chargetransfer integrals as well as effective charge-transfer integrals for different intermonomer separations are presented. Indeed, as seen in the figure, the values of charge-transfer integrals are quite insensitive to the basis set, provided the spatial overlap is taken into account.

The values of the spatial overlap for different geometrical parameters calculated with the aid of different exchangecorrelation potentials as well as using the Hartree-Fock method are presented in Fig. 2b. As seen in the figure, the values of the spatial overlap calculated with use of different DFT functionals are comparable for all considered geometrical parameters. The values of spatial overlap integral calculated using the HF wavefunction seem to be overestimated and differ substantially from the values determined within the DFT framework.

As seen in Fig. 3 the choice of exchange-correlation potential is quite important as far as the magnitude of charge-transfer integral $(J)$ and effective charge-transfer integral $\left(J_{\text {eff }}\right)$ is concerned. The commonly used conventional exchange-correlation potentials such as BLYP or B3LYP predict much smaller values of charge-transfer integrals than the HF method. The PW91 functional, suggested as the best choice for computations of chargetransfer integrals in $\pi$-conjugated systems in stacked configurations [3, 41], gives comparable results to those determined with the aid of the BLYP and the B3LYP potentials. The values of charge-transfer integrals predicted by long-range corrected functionals, namely CAM-B3LYP and LC-BLYP, lie between HF and conventional DFT results (see Fig. 3). It was shown by Peach and co-workers that long-range corrected functionals improve substantially upon their traditional counterparts as far as excitation energies to charge-transfer states are concerned [17]. It is a particularly notable observation for the Coulomb-attenuat- 
a)
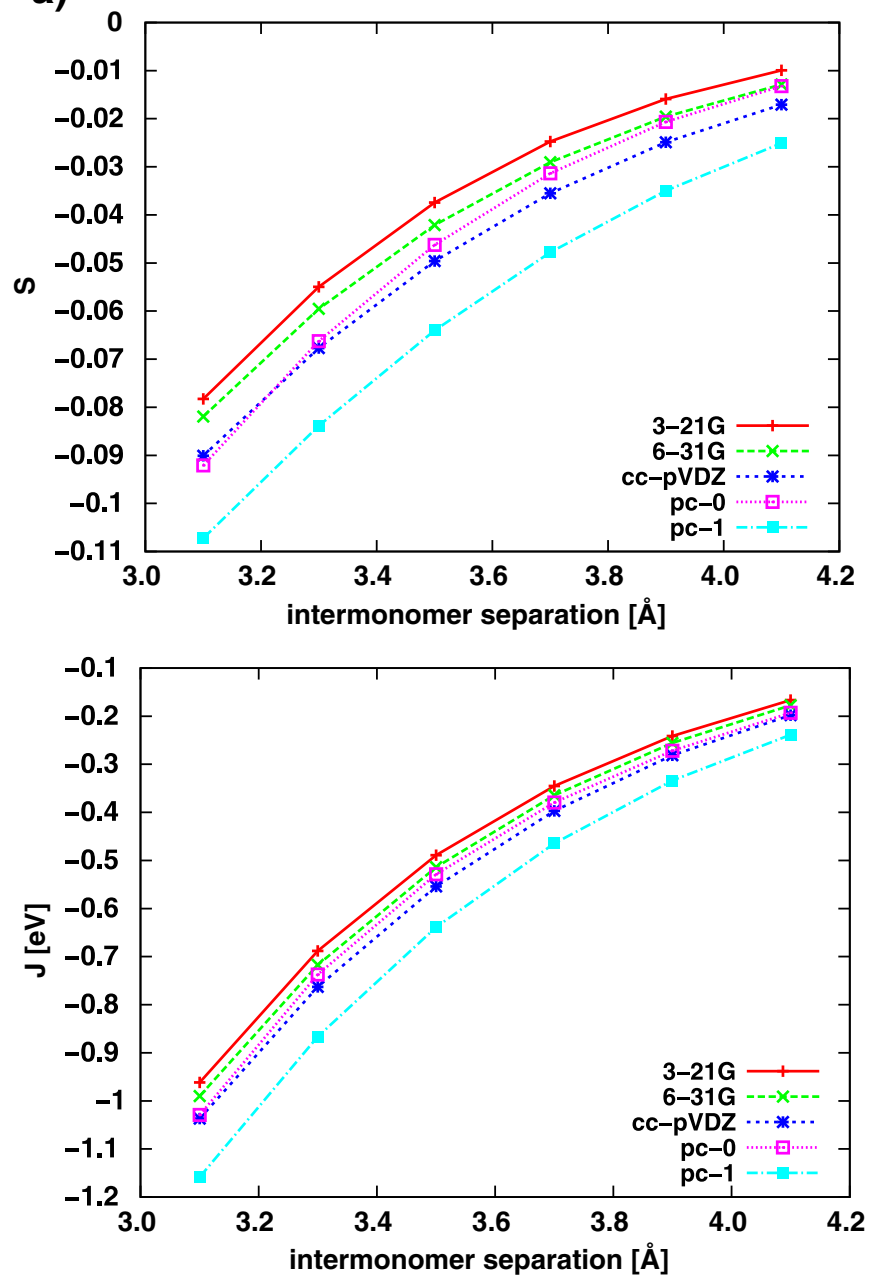

b)
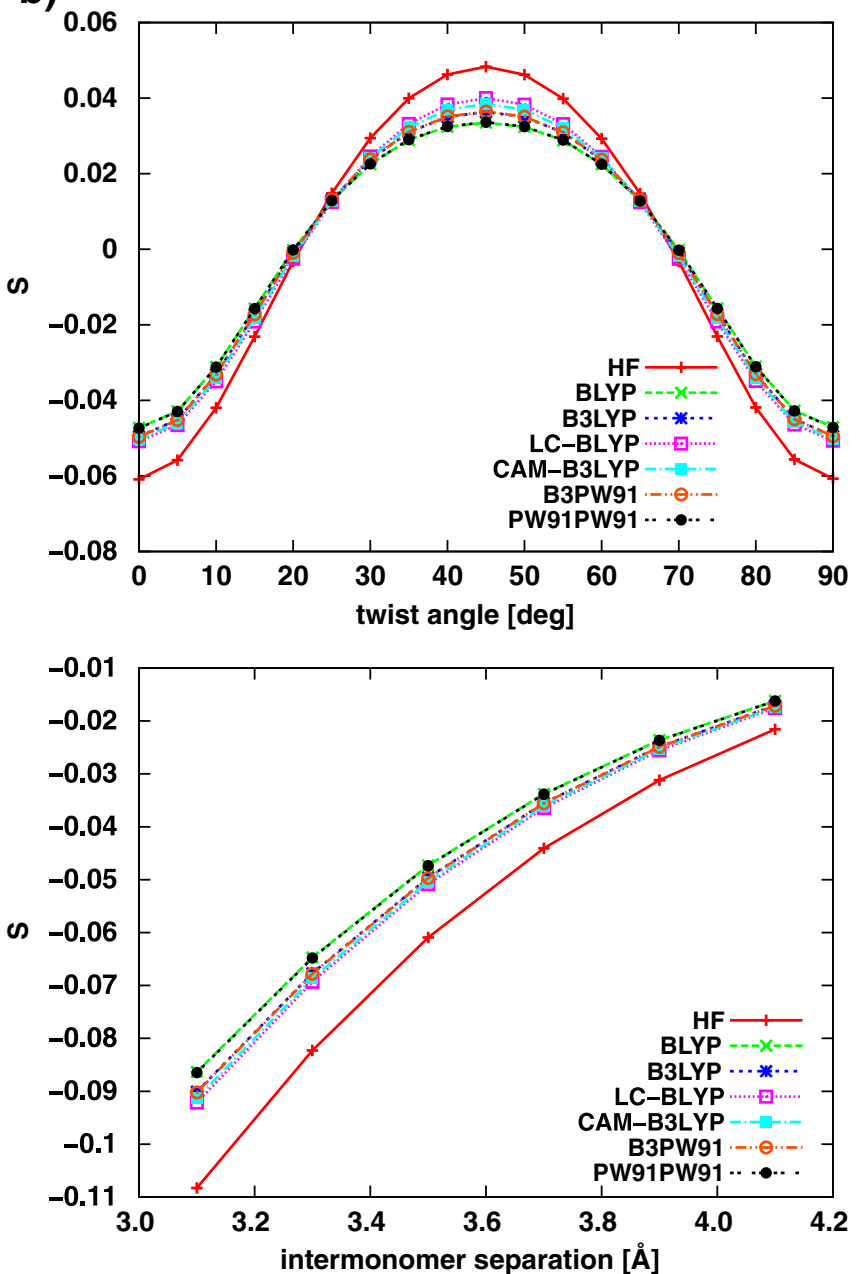

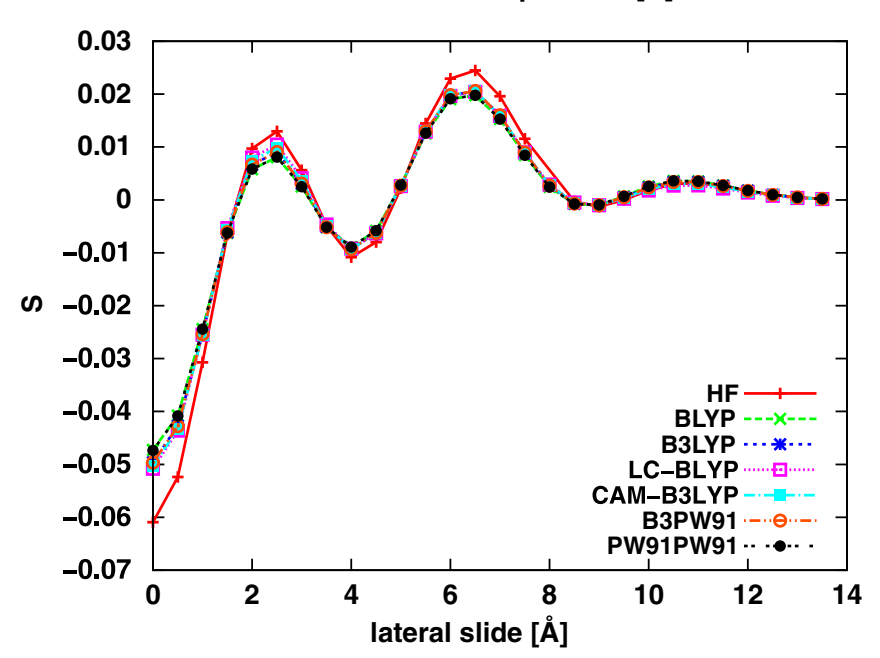

geometrical parameters calculated using various exchange-correlation

potentials and the HF method. The cc-pVDZ basis set was used
Fig. 2 (a) Spatial overlap integrals $(S)$, charge-transfer integrals $(J)$ and effective charge-transfer integrals $\left(J_{e f f}\right)$ computed using the B3LYP functional. (b) Dependence of the spatial overlap on the 

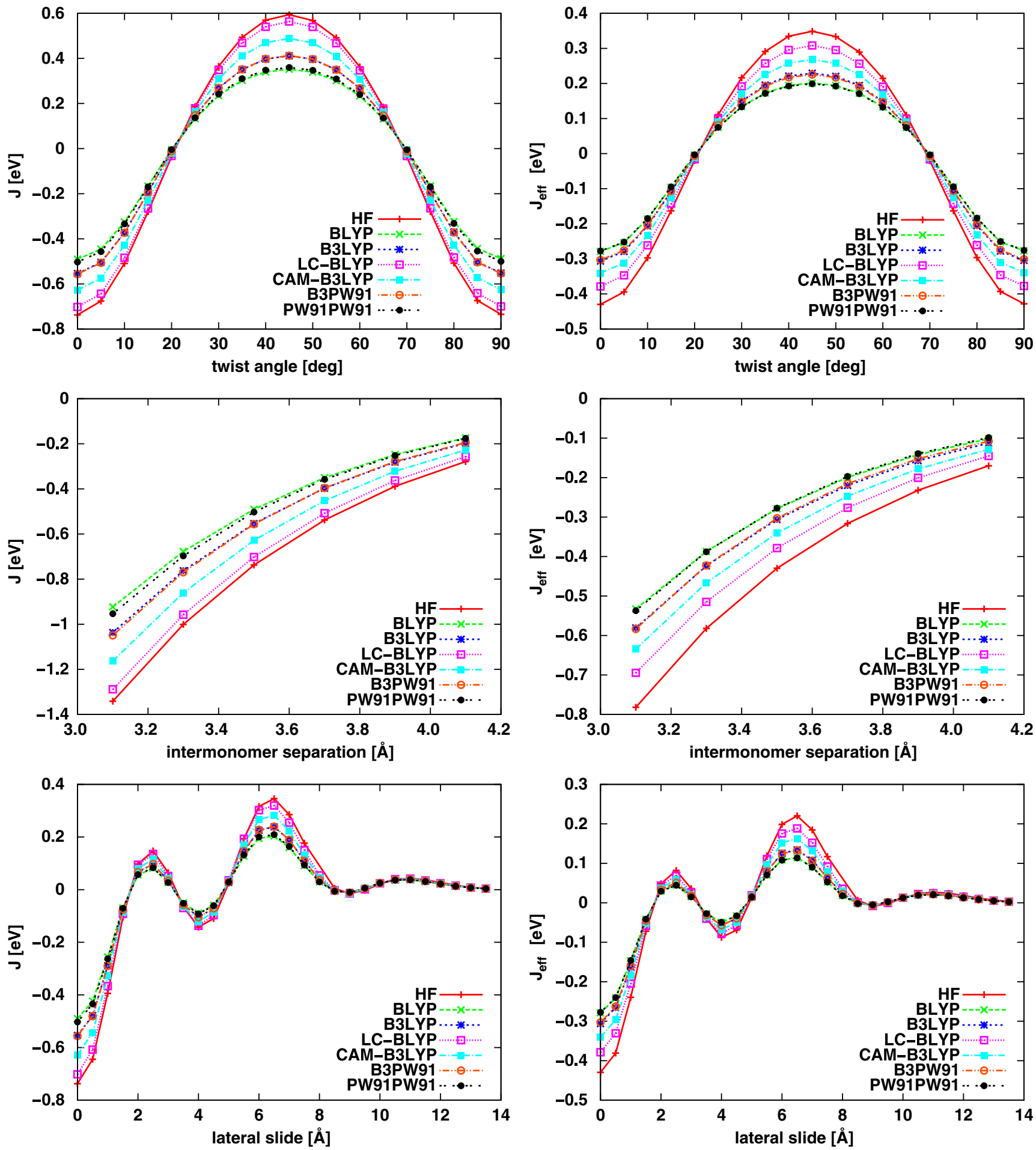

Fig. 3 The dependence of the charge-transfer integral $\left(J\right.$, left side) and the effective charge-transfer integral $\left(J_{\text {eff }}\right.$, right side) on the geometrical parameters for different DFT functionals using the cc-pVDZ basis set

ed model (CAM-B3LYP). Since both quantities in principle might be similar in nature, the LRC potentials should give better results also in the case of charge-transfer integrals. For this reason, with a bit of scepticism due to the lack of more solid quantitative basis, we use the CAM-B3LYP potential as a reference. We conclude that conventional DFT functionals underestimate the values of charge-transfer integrals in comparison with their LRC counterparts. 
One of the theories most commonly used for describing charge transport in organic system is the one proposed by Marcus [44, 45]. In this approach, the hopping rate constant between sites $i$ and $j$ is defined as:

$k_{i j}=\frac{2 \pi\left|J_{i j}\right|^{2}}{h} \sqrt{\frac{\pi}{2 k_{B} \lambda T}} \exp \left(-\frac{\left(\lambda+\varepsilon_{j}-\varepsilon_{i}\right)^{2}}{4 \lambda k_{B} T}\right)$,

where $h, k_{B}$ and $T$ are Planck constant, Boltzmann constant and temperature, respectively. $\varepsilon_{i}$ and $\varepsilon_{j}$ denote energies of the charge carrier localized on the sites $i$ and $j$ and $\lambda$ stands for reorganization energy $[4,13,46]$. In order to estimate the charge carrier mobility in the system without structural disorder (assuming that each hopping rate is the same) one can use the relation $[47,48]$ :

$\mu=\frac{e}{k_{B} T} k_{i j} a^{2}$

where $e$ is elementary charge, and $a$ denotes distance between molecular sites. For the studied phthalo-cyanine dimers, the internal reorganization energy calculated at the $\mathrm{B} 3 \mathrm{LYP} / \mathrm{cc}-\mathrm{pVDZ}$ level of theory is $0.043 \mathrm{eV}$, which is similar to the results presented in literature $[13,49,50]$. For the intermolecular distance $3.5 \AA$, the rotation angle 0 and lateral slide $1.5 \AA$ (this is the structure similar to the crystal structure of the phthalocyanine) the charge-transfer integrals calculated with B3LYP and CAM-B3LYP functional are $-0.16 \mathrm{eV}$ and $-0.18 \mathrm{eV}$ respectively. The charge carrier mobility values calculated from Eq. (11) for this two charge-transfer integrals are $3.9 \mathrm{~cm}^{2} / \mathrm{Vs}$ and $4.9 \mathrm{~cm}^{2} /$ Vs. Thus, one can easily see, that DFT functional has a significant influence on the mobility value. A close look at Fig. 2 leads to the conclusion that for certain areas of conformational space the differences might be even higher.

A comparison of the effective charge-transfer integrals calculated using Eq. (3) and the charge-transfer integrals determined from the energy splitting (Eq. (9)) is shown in Table 1. The data show that the differences in the values of charge-transfer integrals calculated based on the two approaches are insignificant and do not exceed a few thousandths of eV. At first glance, it appears that it is sufficient to employ less accurate method, based on the energy splitting in dimer with assumption of zero spatial overlap, to compute $J$ between molecules in $\pi$ interacting system. However, as it has already been mentioned, it is important to include spatial overlap in calculations of charge-transfer integrals from the definition. Otherwise, the values of $J$ might strongly depend on the size of the basis set used in calculations. The other drawback of the method based on energy splitting in dimer is the lack of information about the sign of charge-transfer integral.
Table 1 The absolute values of effective charge transfer integrals ( $\mid J_{\text {eff }}$, given in eV) computed with the aid of Eqs. (3)-(6) and the charge transfer integrals calculated using energy splitting in dimer approach $(|\Delta / 2|)$. The cc-pVDZ basis set was employed in all calculations. $R$ is the intermolecular distance

\begin{tabular}{|c|c|c|c|c|c|c|}
\hline & \multicolumn{2}{|l|}{ B3LYP } & \multicolumn{2}{|l|}{ BLYP } & \multicolumn{2}{|l|}{$\underline{\mathrm{HF}}$} \\
\hline & $\left|J_{e f f}\right|$ & $\Delta / 2$ & $\left|J_{e f f}\right|$ & $\Delta / 2$ & $\left|J_{e f f}\right|$ & $\Delta / 2$ \\
\hline \multicolumn{7}{|c|}{$\mathrm{R}[\AA]$} \\
\hline 3.1 & 0.5809 & 0.5859 & 0.5327 & 0.5372 & 0.7820 & 0.7898 \\
\hline 3.3 & 0.4235 & 0.4254 & 0.3870 & 0.3887 & 0.5820 & 0.5850 \\
\hline 3.5 & 0.3061 & 0.3068 & 0.2787 & 0.2793 & 0.4297 & 0.4309 \\
\hline 3.7 & 0.2198 & 0.2200 & 0.1995 & 0.1996 & 0.3161 & 0.3165 \\
\hline 3.9 & 0.1572 & 0.1571 & 0.1421 & 0.1420 & 0.2320 & 0.2320 \\
\hline \multirow[t]{3}{*}{4.1} & 0.1125 & 0.1124 & 0.1015 & 0.1014 & 0.1701 & 0.1699 \\
\hline & \multicolumn{2}{|c|}{ CAM-B3LYP } & \multicolumn{2}{|c|}{ LC-BLYP } & \multicolumn{2}{|c|}{ B3PW 91} \\
\hline & $\left|J_{e f f}\right|$ & $\Delta / 2$ & $\left|J_{e f f}\right|$ & $\Delta / 2$ & $\left|J_{e f f}\right|$ & $\Delta / 2$ \\
\hline \multicolumn{7}{|c|}{$\mathrm{R}[\AA]$} \\
\hline 3.1 & 0.6344 & 0.6399 & 0.6944 & 0.7006 & 0.5836 & 0.5886 \\
\hline 3.3 & 0.4667 & 0.4689 & 0.5149 & 0.5174 & 0.4226 & 0.4245 \\
\hline 3.5 & 0.3404 & 0.3412 & 0.3785 & 0.3796 & 0.3030 & 0.3037 \\
\hline 3.7 & 0.2467 & 0.2469 & 0.2763 & 0.2767 & 0.2153 & 0.2155 \\
\hline 3.9 & 0.1781 & 0.1781 & 0.2005 & 0.2007 & 0.1523 & 0.1522 \\
\hline 4.1 & 0.1284 & 0.1283 & 0.1451 & 0.1452 & 0.1076 & 0.1075 \\
\hline
\end{tabular}

However, if the knowledge of the sign is important, it can be subsequently determined from the bonding-antibonding character of the interaction between the corresponding orbitals [51].

\section{Conclusions}

The primary aim of the present study was to evaluate the performance of commonly employed conventional exchange-correlation potentials that are used to compute charge-transfer integrals. In doing so, we apply the recently proposed Coulomb-attenuated model as a reference as this approach is proven to be very successful in predicting excitation energies to charge-transfer states. It is shown that for certain areas of conformational space in phthalocyanine dimer the differences in values of charge-transfer integrals between the conventional schemes and the CAM-B3LYP functional in values of charge-transfer integrals might be quite significant. The same is revealed for triphenylene dimer [52]. As a result, the values of charge carrier mobilities estimated using Marcus formula might differ by $20 \%$ and more. Likewise, theoretical predictions of peaks intensity in electro-absorption spectrum of molecular crystals and molecular aggregates $[53,54]$ might be determined to a large extent by the accuracy of charge-transfer integrals (Kulig W, Petelenz P, (2010). Private communication). We have also confirmed the findings reported by other authors 
[41] that the size of the basis set used in calculations of charge-transfer integrals plays only a minor role provided the spatial overlap is included in the theoretical model.

Acknowledgments This work was supported by computational grants from Wroclaw Center for Networking and Supercom- puting (WCSS) and ACK Cyfronet. Work in the USA was supported by the HRD-0833178 grant. One of the authors (RZ) would like to acknowledge support from a grant from Iceland, Liechtenstein and Norway through the EEA Financial Mechanism - Scholarship and Training Fund. Financial support from Wroclaw University of Technology and the Czech Science Foundation (Project No. P205/ 10/2280) and the European Commission through the Human Potential Programme (Marie-Curie RTN BIMORE, Project No. MRTN-CT2006-035859) is also acknowledged.

Open Access This article is distributed under the terms of the Creative Commons Attribution Noncommercial License which permits any noncommercial use, distribution, and reproduction in any medium, provided the original author(s) and source are credited.

\section{References}

1. Brédas JL, Calbert JP, da Silva Filho DA, Cornil J (2002) PNAS 99(9):5804

2. Wang L, Nan G, Yang X, Peng Q, Li Q, Shuai Z (2010) Chem Soc Rev 39:423

3. Senthilkumar K, Grozema FC, Bickelhaupt FM, Siebbeles LDA (2003) J Chem Phys 119:9809

4. Newton MD (1991) Chem Rev 91:767

5. Cheung DL, Troisi A (2008) Phys Chem Chem Phys 10:5941

6. Bassler H (1993) Phys Stat Sol (b) 175:15

7. Toman P, Nespurek S, Bartkowiak W (2009) Mater Sci Poland 27:797

8. Hreha RD, George CP, Haldi A, Domercq B, Malagoli M, Barlow S, Brédas J-L, Kippelen B, Marder SR (2003) Adv Funct Mater 13:967

9. Yang B, Kim S-K, Xu H, Park Y-I, Zhang H, Gu C, Shen F, Wang C, Liu D, Liu X, Hanif M, Tang S, Li W, Li F, Shen J, Park J-W, Ma Y (2008) Chem Phys Chem 9:2601

10. Gao H, Zhang H, Mo R, Sun S, Su Z-M, Wang Y (2009) Synth Met 159:1767

11. Zbiri M, Johnson MR, Kearley GJ, Mulder FM (2010) Theor Chem Acc 125:445

12. Delgado MCR, Kim EG, da DA, Filho S, Brédas JL (2010) J Am Chem Soc 132:3375

13. Lee C, Sohlberg K (2010) Chem Phys 367:7

14. Chen S, Ma J (2009) J Comp Chem 30:1959

15. Baerends EJ, Gritsenko OV (1997) J Phys Chem A 101:5383

16. Tozer DJ, Amos RD, Handy NC, Roos BO, Serrano-Andres L (1999) Mol Phys 97:859

17. Peach MJG, Benfield P, Helgaker T, Tozer DJ (2008) J Chem Phys 128:044118

18. Kamiya M, Sekino H, Tsuneda T, Hirao K (2005) J Chem Phys $122: 234111$

19. Kirtman B, Bonness S, Ramirez-Solis A, Champagne B, Matsumoto H, Sekino H (2008) J Chem Phys 128:114108
20. Jacquemin D, Perpéte EA, Scalmani G, Frisch MJ, Kobayashi R, Adamo C (2007) J Chem Phys 126:144105

21. Loboda O, Zaleśny R, Avramopoulos A, Luis JM, Kirtman B, Tagmatarchis N, Reis H, Papadopoulos MG (2009) J Phys Chem A 113:1159

22. Jacquemin D, Perpète EA, Medved M, Scalmani G, Frisch MJ, Kobayashi R, Adamo C (2007) J Chem Phys 126:191108

23. Hammond JR, Kowalski K (2009) J Chem Phys 130:194108

24. Limacher PA, Mikkelsen KV, Lüthi HP (2009) J Chem Phys 130:194114

25. Zaleśny R, Wójcik G, Mossakowska I, Bartkowiak W, Avramopoulos A, Papadopoulos MG (2009) J Mol Struct THEOCHEM 901:46

26. Casida ME, Salahub DR (2000) J Chem Phys 113:8918

27. Cai ZL, Crossley MJ, Reimers JR, Kobayashi R, Amos RD (2006) J Phys Chem B 110:15624

28. Silva D, Krawczyk P, Bartkowiak W, Mendonca CR (2009) J Chem Phys 131:244516

29. Rostov IV, Amos RD, Kobayashi R, Scalmani G, Frisch MJ (2010) J Phys Chem B 114:5547

30. Iikura H, Tsuneda T, Yanai T, Hirao K (2001) J Chem Phys 115:3540

31. Yanai T, Tew DP, Handy NC (2004) Chem Phys Lett 393:51

32. Frisch MJ et al (2009) Gaussian 09 Revision A.1. Gaussian Inc. Wallingford CT

33. Facchetti A (2007) Mater Today 10(3):28

34. Newman CR, Frisbie CD, da Silva Filho DA, Brédas JL, Ewbank PC, Mann KR (2004) Chem Mater 16:4436

35. Toman P, Nespurek S, Yakushi K (2002) J Porphyr Phthalocyanines 6:556

36. Shirota Y, Kageyama H (2007) Chem Rev 107:953

37. Sergeyev S, Pisula W, Geerts YH (2007) Chem Soc Rev 36:1902

38. Mikolajczyk MM, Toman P, Bartkowiak W (2010) Chem Phys Lett 485:253

39. Cornil J, Beljonne D, Calbert JP, Brédas JL (2001) Adv Mater 13 (14): 1053

40. Huang J, Kertesz M (2004) Chem Phys Lett 390:110

41. Huang J, Kertesz M (2005) J Chem Phys 122:234707

42. Dunning TH (1989) J Chem Phys 90:1007

43. Jensen F (2001) J Chem Phys 115:9113

44. Marcus RA (1993) Rev Mod Phys 65:599

45. Barbara PF, Meyer TJ, Ratner MA (1996) J Phys Chem 100:13148

46. Hutchison GR, Ratner MA, Marks TJ (2005) J Am Chem Soc 127:2339

47. Grozema FC, Siebbeles LDA (2008) Int Rev Phys Chem 27:87

48. Berlin YA, Grozema FC, Siebbeles LDA, Ratner MA (2008) J Phys Chem C 112:10988

49. Tant J, Geerts YH, Lehmann M, Cupere VD, Zucchi G, Laursen BW, Bjornholm T, Lemaur V, Marcq V, Burquel A, Hennebicq E, Gardebien F, Viville P, Beljonne D, Lazzaroni R, Cornil J (2005) J Chem Phys B 109:20315

50. Chang YC, Chao I (2010) J Phys Chem Lett 1:116

51. Seo D, Hoffmann R (1999) Theor Chem Acc 102:23

52. Mikołajczyk M, Zaleśny R, Czyznikowska Z, Bartkowiak W, Toman P, Leszczynski J (2009). Unpublished results

53. Stradomska A, Petelenz P (2006) Mol Phys 104:2063

54. Petelenz P (2004) Org Electron 5:115 